\title{
Reflection and transmission of solar light by clouds: asymptotic theory
}

\author{
A. A. Kokhanovsky ${ }^{1}$ and T. Nauss ${ }^{2}$ \\ ${ }^{1}$ Institute of Remote Sensing, University of Bremen, O. Hahn Allee 1, 28334 Bremen, Germany \\ ${ }^{2}$ Laboratory of Climatology and Remote Sensing, Marburg University, Deutschhausstr. 10, 35032 Marburg, Germany
}

Received: 22 May 2006 - Published in Atmos. Chem. Phys. Discuss.: 31 August 2006

Revised: 2 November 2006 - Accepted: 30 November 2006 - Published: 11 December 2006

\begin{abstract}
The authors introduce a radiative transfer model CLOUD for reflection, transmission, and absorption characteristics of terrestrial clouds and discuss the accuracy of the approximations used within the model. A Fortran implementation of CLOUD is available for download. This model is fast, accurate, and capable of calculating multiple radiative characteristics of cloudy media including the spherical and plane albedo, reflection and transmission functions, absorptance as well as global and diffuse transmittance. The approximations are based on the asymptotic solutions of the radiative transfer equations valid at cloud optical thicknesses larger than 5.

While the analytic part of the solutions is treated in the code in an approximate way, the correspondent reflection function (RF) of a semi-infinite water cloud $R_{\infty}$ is calculated using numerical solutions of the radiative transfer equation in the assumption of Deirmendjian's cloud C1 model. In the case of ice clouds, the fractal ice crystal model is used. The resulting values of $R_{\infty}$ with respect to the viewing geometry are stored in a look-up table (LUT).

The results obtained are of importance for quick estimations of main radiative characteristics of clouds and also for the solution of inverse problems.
\end{abstract}

\section{Introduction}

Clouds occupy more than $60 \%$ of the sky at any given time and are main regulators of weather and climate. Because the study of cloud properties on a global scale is of significant importance, many satellite cloud retrieval algorithms have been developed over the past decades whereupon most of these are based on so-called Look-Up Tables (LUTs) for the reflection function of clouds (e.g. Nakajima and King,

Correspondence to: A. A. Kokhanovsky

(alexk@iup.physik.uni-bremen.de)
1990). Kokhanovsky et al. (2003) have proposed an algorithm which is based on the analytical asymptotic solutions of the radiative transfer equations valid for optically thick clouds with a cloud optical thickness $\tau$ larger than approximately 5 and a single scattering albedo $\omega_{0}$ larger than 0.98 . Since the latter property limits the application to weakly absorbing clouds, we propose a new technique to overcome the limitation of small values of $\omega_{0}$ which is of special importance for ice cloud remote sensing when the size of the scattering particles and therefore the absorption of radiation in the cloud can be quite large. Moreover, the 3.6 to $3.9 \mu \mathrm{m}$ channels available on many satellite sensors are no longer excluded from the potential range of applications.

The new technique is based on simple equations that can be used for approximate analytical calculations of the reflection functions and light fluxes in cloudy atmospheres. Therefore, they enable the simplification of existing satellite and ground-based cloud retrieval algorithms while errors in the computation of the reflection function are generally smaller than errors related to uncertainties in the forward model and errors due to calibration uncertainties of optical instruments.

In the following section, a system of equations that accounts for multiple light scattering is presented, which can be used for the forward modeling of cloud optical properties. The accuracy of these analytical equations with respect to cloud reflection and transmission characteristics is discussed in Sect. 3.

The presented results can be used for the rapid estimates of various radiative cloud characteristics and also for the speeding up cloud retrieval algorithms.

\section{General equations}

It is well known that reflection $R_{A}\left(\mu_{0}, \mu, \phi\right)$ and transmission $T_{A}\left(\mu_{0}, \mu\right)$ functions of optically thick homogeneous plane-parallel light scattering layers over a Lambertian

Published by Copernicus GmbH on behalf of the European Geosciences Union. 
surface with albedo $A$ can be presented in the following analytical forms (van de Hulst, 1980; Kokhanovsky, 2006):

$$
\begin{aligned}
& R_{A}\left(\mu_{0}, \mu, \phi\right)=R\left(\mu_{0}, \mu, \phi\right)+\frac{A t_{d}\left(\mu_{0}\right) t_{d}(\mu)}{1-A r_{s}}, \\
& R\left(\mu_{0}, \mu, \phi\right)=R_{\infty}\left(\mu_{0}, \mu, \phi\right)-T\left(\mu_{0}, \mu\right) e^{-k \tau}, \\
& T_{A}\left(\mu_{0}, \mu\right)=T\left(\mu_{0}, \mu\right)+\frac{A t_{d}\left(\mu_{0}\right) r_{p}(\mu)}{1-A r_{s}}, \\
& T\left(\mu_{0}, \mu\right)=\frac{m e^{-k \tau}}{1-l^{2} e^{-2 k \tau}} K\left(\mu_{0}\right) K(\mu) .
\end{aligned}
$$

Here $R \equiv R_{A}(A=0), T \equiv T_{A}(A=0)$, the pair $\left(\mu_{0}, \mu\right)$ gives the cosines of the incidence and observation angles, $\phi$ is the relative azimuth, $t_{d}\left(\mu_{0}\right)$ is the diffuse transmittance of a layer under illumination along the direction $\vartheta_{0}=\arccos \left(\mu_{0}\right), r_{s}$ is the spherical albedo, $r_{p}(\mu)$ is the plane albedo for the diffuse illumination conditions and an observation along the direction specified by the viewing zenith angle $\vartheta=\arccos \mu . \quad R_{\infty}\left(\mu_{0}, \mu, \phi\right)$ is the reflection function of a semi-infinite scattering layer having the same local optical characteristics (e.g., the same single scattering albedo $\omega_{0}$ and the same phase function $p(\theta)$ with scattering angle $\theta$ ) as the finite layer currently under study. The functions $r_{p}(\mu), t_{d}(\mu)$ and $r_{s}$ are defined as

$$
\begin{aligned}
& r_{p}(\mu)=2 \int_{0}^{1} \bar{R}\left(\mu_{0}, \mu\right) \mu_{0} d \mu_{0}, \\
& t_{d}(\mu)=2 \int_{0}^{1} \bar{T}\left(\mu_{0}, \mu\right) \mu_{0} d \mu_{0}, \\
& r_{s}=2 \int_{0}^{1} r_{p}(\mu) \mu d \mu,
\end{aligned}
$$

with $\quad \bar{R}\left(\mu_{0}, \mu\right)=\frac{1}{2 \pi} \int_{0}^{2 \pi} R\left(\mu_{0}, \mu, \phi\right) d \phi$ and

$\bar{T}\left(\mu_{0}, \mu\right)=\frac{1}{2 \pi} \int_{0}^{2 \pi} R\left(\mu_{0}, \mu, \phi\right) d \phi$. The constants $(k, l, m)$ and the escape function $K(\mu)$ do not depend on $\tau$ and can be obtained from the solution of integral equations as described by van de Hulst (1980), Wauben (1992), and Kokhanovsky (2006). Expressions for $r_{p A}(\mu), t_{d A}(\mu)$ and $r_{s A}$ for arbitrary surface albedo values $\mathrm{A}$ can be derived from Eqs. (1), (2) with account for the definitions in Eq. (3). Namely it follows:

$$
\begin{aligned}
& r_{p A}(\mu)=r_{p}(\mu)+\frac{A t^{2} K(\mu)}{n\left(1-A r_{s}\right)}, \\
& r_{p}(\mu)=r_{p \infty}(\mu)-l t n^{-1} K(\mu) e^{-k \tau}, \\
& r_{s A}=r_{s}+\frac{A t^{2}}{1-A r_{s}}, \\
& r_{s}=r_{s \infty}-l t e^{-k \tau},
\end{aligned}
$$

$$
\begin{aligned}
t_{d A}(\mu) & =t_{d}(\mu)+\frac{A t r_{p}(\mu)}{1-A r_{s}}, \\
t_{d}(\mu) & =t n^{-1} K(\mu),
\end{aligned}
$$

where $t$ is the global transmittance defined by (see Eqs. 2, 3)

$$
t \equiv 2 \int_{0}^{1} t_{d}(\mu) \mu d \mu=\frac{m n^{2} e^{-k \tau}}{1-l^{2} e^{-2 k \tau}}
$$

and

$n=2 \int_{0}^{1} K(\mu) \mu d \mu$.

Note that it follows from Eqs. (3) and (6-8):

$t_{A}=\frac{t}{1-A r_{s}}$

A summary of the main equations can be found in Table 1.

The radiative characteristics $r_{p}(\mu), t_{d}(\mu), r_{s}, t$ as well as $R\left(\mu_{0}, \mu, \phi\right)$ and $T\left(\mu_{0}, \mu\right)$ can be measured directly. Therefore, equations shown above can be of importance for the interpretation of correspondent experiments. For the use of the analytical Eqs. (1), (2), (4-7), (9) at arbitrary $\omega_{0}$ and $p(\theta)$, several parameters $\left(k, l, m, n, r_{s}\right)$ and also functions $K(\mu), R_{\infty}\left(\mu_{0}, \mu, \phi\right)$ and $r_{p \infty}(\mu)$ have to be derived. The problem is simplified when $\omega_{0}=1$. Then it follows: $k=m=0, l=n=r_{s \infty}=r_{p \infty}(\mu)=1$. For the general case parameters $k, l, m, n, r_{s} \infty$ can be parameterized as follows (van de Hulst, 1974; King and Harshvardan, 1986):

$k=\left(\sqrt{3} s-\frac{(0.985-0.253 s) s^{2}}{6.464-5.464 s}\right)\left(1-\omega_{0} g\right)$,

$l=\frac{(1-s)(1-0.681 s)}{1+0.792 s}$,

$m=(1+1.537 s) \ln \left(\frac{1+1.8 s-7.087 s^{2}+4.74 s^{3}}{(1-0.819 s)(1-s)^{2}}\right)$,

$n=\sqrt{\frac{(1-s)(1+0.414 s)}{1+1.888 s}}$,

$r_{s \infty}=\frac{(1-s)(1-0.139 s)}{1+1.17 s}$,

where

$s=\sqrt{\frac{1-\omega_{0}}{1-\omega_{0} g}}$

is the similarity parameter and

$g=\frac{1}{4} \int_{0}^{\pi} p(\theta) \sin (2 \theta) d \theta$ 
Table 1. Radiative transfer characteristics of optically thick layers.

\begin{tabular}{lll}
\hline Radiative characteristic & $A=0$ & $A \neq 0$ \\
\hline $\begin{array}{l}\text { Transmission } \\
\text { function }\end{array}$ & $T\left(\mu_{0}, \mu\right)=t n^{-2} K\left(\mu_{0}\right) K(\mu)$ & $\begin{array}{l}T_{A}\left(\mu_{0}, \mu\right)=t n^{-2} K\left(\mu_{0}\right) K(\mu)+ \\
\frac{A t n^{-1} K\left(\mu_{0}\right) r_{d}(\mu)}{1-A r_{s}}\end{array}$ \\
$\begin{array}{l}\text { Diffuse } \\
\text { transmittance }\end{array}$ & $t_{d}(\mu)=t n^{-1} K(\mu)=t n^{-1} K(\mu)+\frac{A t r_{p}(\mu)}{1-A r_{s}}$ \\
$\begin{array}{l}\text { Global } \\
\text { transmittance }\end{array}$ & $t=\frac{m n^{2} e^{-k \tau}}{1-l^{2} e^{-2 k \tau}}$ & $t_{A}=\frac{m n^{2} e^{-k \tau}}{\left(1-l^{2} e^{-2 k \tau}\right)\left(1-A r_{s}\right)}$ \\
Reflection function & $R\left(\mu_{0}, \mu, \phi\right)=R_{\infty}\left(\mu_{0}, \mu, \phi\right)$ & $R_{A}\left(\mu_{0}, \mu, \phi\right)=R_{\infty}\left(\mu_{0}, \mu, \phi\right)$ \\
& $-l t n^{-2} K\left(\mu_{0}\right) K(\mu) e^{-k \tau}$ & $-l t n^{-2} K\left(\mu_{0}\right) K(\mu) e^{-k \tau}$ \\
& $+\frac{A t^{2} n^{-2} K\left(\mu_{0}\right) K(\mu)}{1-A r_{s}}$ \\
Plane albedo & $r_{p}(\mu)=r_{p \infty}(\mu)-l t n^{-1} K(\mu) e^{-k \tau}$ & $r_{p A}=r_{p \infty}(\mu)-l t n^{-1} K(\mu)+\frac{A t^{2} n^{-1} K(\mu)}{1-A r_{s}}$ \\
Spherical albedo & $r_{s}=r_{s \infty}-l t e^{-k \tau}$ & $r_{s A}=r_{s \infty}(\mu)-l t e^{-k \tau}+\frac{A t^{2}}{1-A r_{s}}$ \\
\hline
\end{tabular}

is the asymmetry parameter.

Functions $K(\mu), R_{\infty}\left(\mu_{0}, \mu, \phi\right)$ and $r_{p \infty}(\mu)$ can not be parameterized in terms of the similarity parameter alone. Therefore, LUTs of $K(\mu)$ for different values of $\mu$ and $\omega_{0}$ have been calculated using the Heney-Greenstein phase function (van de Hulst, 1980) with an asymmetry parameter $g=0.85$ that is typical for water clouds. This can be done since both functions $K(\mu)$ and $r_{p \infty}(\mu)$ show only a weak dependence on the phase function and even on $g$ for a fixed value of the similarity parameter (Kokhanovsky, 2006). For these calculations, a radiative transfer code developed by Wauben (1992) has been used which is based on van de Hulst's asymptotic fitting technique (van de Hulst, 1980). The LUT for $r_{p \infty}(\mu)$ has been prepared for a wavelength of $0.65 \mu \mathrm{m}$ and Deirmendjian's cloud C1 model phase function (Kokhanovsky, 2006) using a radiative transfer code developed by Mishchenko et al. (1999). The final LUTs are represented in terms of $\mu$ and $s$ so they can be applied at arbitrary values of $g$ and $\omega_{0}$. Due to the reciprocity principle (van de Hulst, 1980), functions $K(\mu)$ and $r_{p \infty}(\mu)$ are identical to $K\left(\mu_{0}\right)$ and $r_{p \infty}\left(\mu_{0}\right)$ for the media under consideration.

The system of equations and LUTs specified above can be used to calculate all radiative properties of an optically thick layer except $R\left(\mu_{0}, \mu, \phi\right)$. In order to compute $R\left(\mu_{0}, \mu, \phi\right)$, the reflection function of a semi-infinite layer $R_{\infty}\left(\mu_{0}, \mu, \phi\right)$ must be determined. It depends on three angular parameters and also on the single scattering albedo and the phase function. Values of $R_{\infty}\left(\mu_{0}, \mu, \phi\right)$ enter the code through look-up tables that have been computed for the wavelength $\lambda=0.65 \mu \mathrm{m}$ and different values of the single scattering albedo using the code developed by Mishchenko et al. (1999). A fixed gamma particle size distribution $f(a)=M a^{6} \exp (-1.5 a)$ with an effective radius of $6 \mu \mathrm{m}$ (ratio of the third to the second moment of $f(a))$ has been assumed; $a$ is the radius of the particles and $M=3^{5} /\left(2^{4} \times 5^{8}\right)$ is a normalization constant $\left(\int_{0}^{\infty} f(a) d a=1\right)$. For the wavelength of $\lambda=0.65 \mu \mathrm{m}$, a refractive index of 1.330683 has been chosen (Kokhanovsky, 2006) resulting in an asymmetry parameter equal to 0.85 .

For ice clouds, the LUT for $R_{\infty}(\lambda=0.65 \mu \mathrm{m})$ is based on the fractal model of scatterers for the phase function described by Mishchenko et al. (1999). A refractive index of 1.311 has been assumed, which leads to an asymmetry parameter of $g=0.7524$ for irregular fractal particles. Due to the large values of $a_{e f}$ for ice crystals, the pre-computed LUTs are almost insensitive to the wavelength used for their calculations.

A Fortran code (CLOUD) performing all calculations according to the equations given above as well as all necessary LUTs can be downloaded from the internet http://www.iup. physik.uni-bremen.de/ alexk. The code is fast, reliable, easy to use, and accurate and therefore enables a rapid estimation of various cloud radiative transfer characteristics.

\section{The accuracy of approximations}

\subsection{Diffuse light characteristics}

The accuracy of the approximations for the plane albedo $r_{p}$, the diffuse transmittance $t_{d}$, and the diffuse absorptance $a_{d}=1-r_{p}-t_{d}$ is shown in Figs. $1-5$ obtained using the exact radiative transfer code SCIATRAN (Rozanov et al., 2005 ) for a typical water cloud phase function calculated at $\lambda=0.65 \mu \mathrm{m}$ and the particle size distribution described above.

Let us consider the obtained results in more details. First of all, one can see that the approximate equations describe the correspondent physical dependencies correctly. It follows 


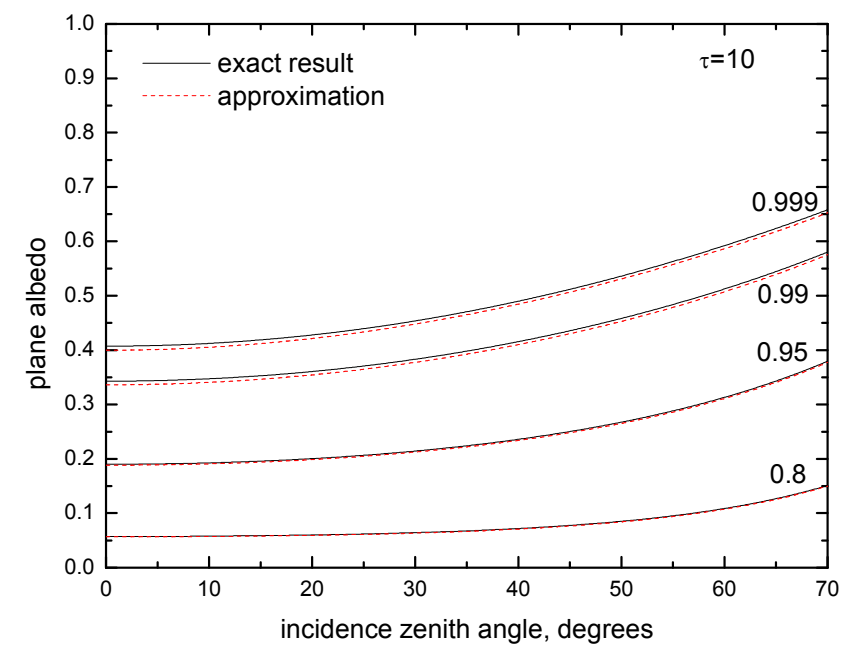

Fig. 1a. Dependence of the plane albedo on the incidence zenith angle for several values of the single scattering albedo and an optical thickness of 10. Exact results are given by black lines. Approximate results are given by red broken lines.

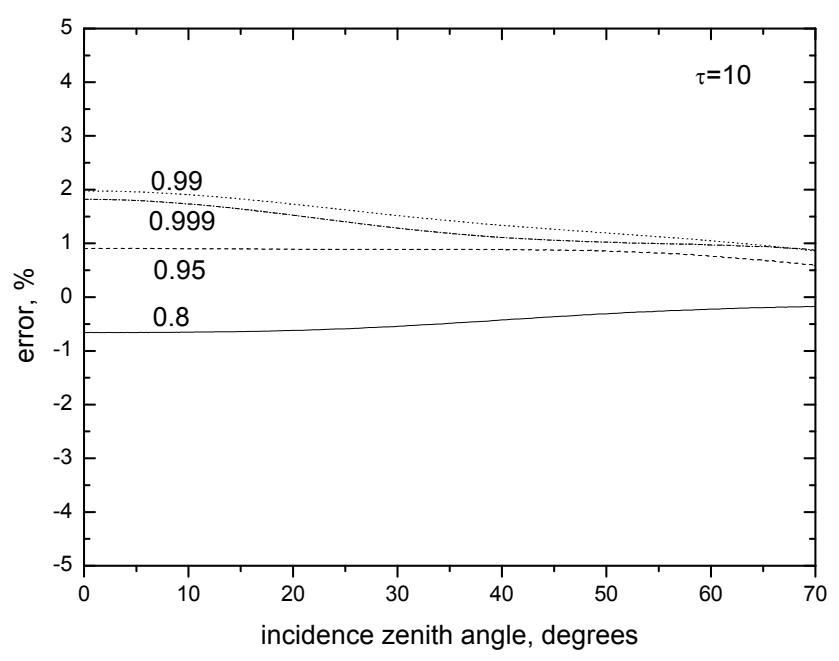

Fig. 1b. Relative error $\delta$ between the approximated values of the plane albedo as a function of the incidence zenith angle obtained from data shown in Fig. 1a.

from Fig. 1a that the plane albedo increases with the incident zenith angle $\vartheta_{0}$ while the diffuse transmittance (Fig. 2) decreases. The reason for this is quite obvious. Light entering the medium under grazing angles has more chances to escape from the medium at the illuminated side. Interestingly, the diffuse absorptance (Fig. 3) is only weakly influenced by the light source angular position. The relative error $\delta=100\left(1-x_{a} / x_{e}\right)\left(x_{a}\right.$ is the approximate value and $x_{e}$ is the exact result derived from SCIATRAN) is very small. For the plane albedo it is smaller than $2 \%$ at $\tau=10$ and $\omega_{0} \geq 0.8$ (see

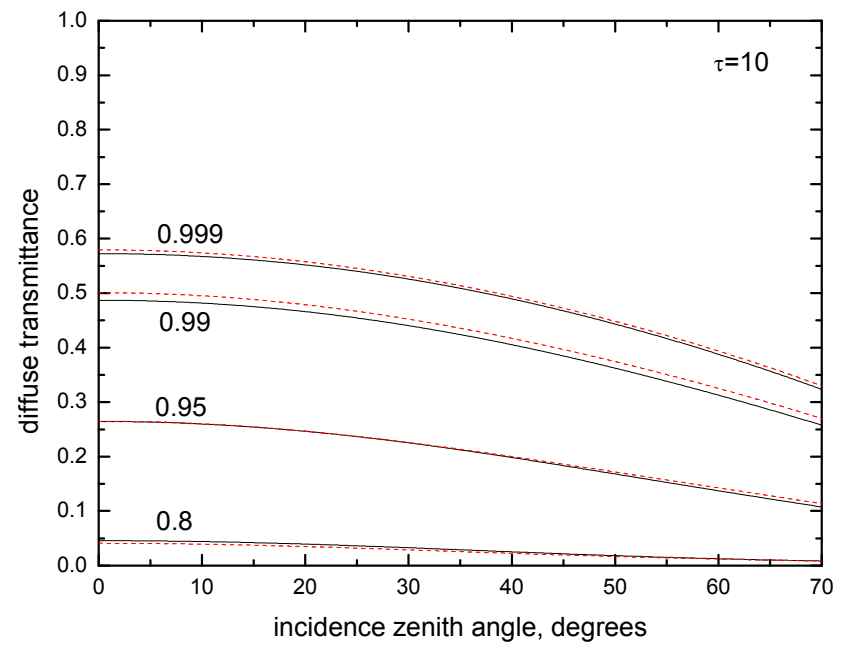

Fig. 2. The same as in Fig. 1a except for the diffuse transmission.

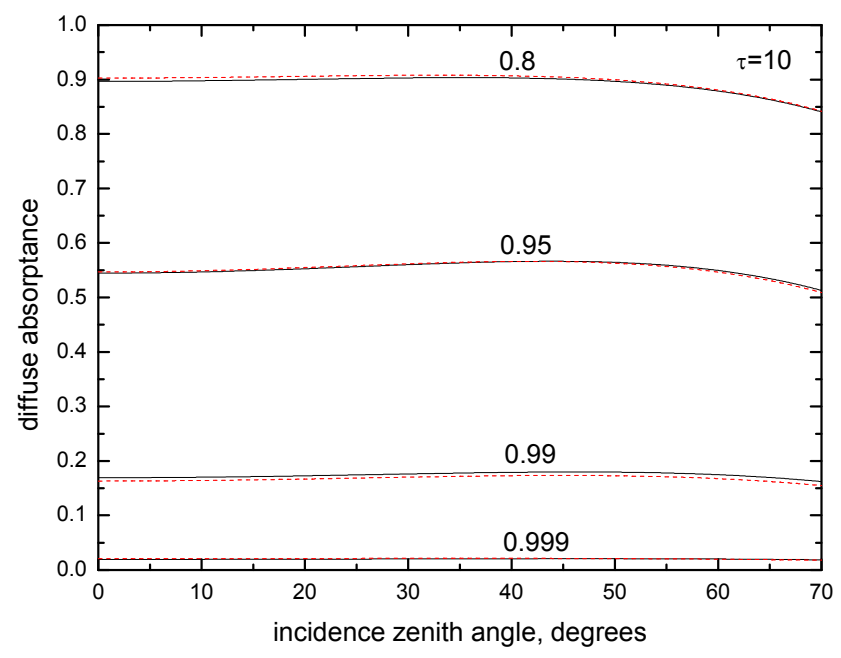

Fig. 3. The same as in Fig. 1a except for the diffuse absorptance.

Fig. 1b). The correspondent $\delta$ for the diffuse transmittance is somewhat larger. It can reach $12 \%$ at $\omega_{0}=0.8$. However, errors are smaller than $6 \%$ for values of the single scattering albedo larger than 0.95 and $\tau \geq 3$. Such an error of the diffuse transmittance will be translated to a much smaller error for the reflection function given by Eq. (1) due to the small influences of the surface term on the satellite signal in the case of thick clouds considered in this study. The error in $a_{d}$ is smaller than $8 \%$ at $\omega_{0} \geq 0.8$ and it is smaller than $5 \%$ at $\omega_{0} \geq 0.95$. Consequently, equations given above can also be used for the estimation of the absorption and heating rates in cloud fields over underlying surfaces with varying albedos.

Figure 4a clearly shows that the approximation for the plane albedo $r_{p}$ can be used for clouds with $\tau \geq 3$ with errors smaller than $5 \%$ (see Fig. $4 \mathrm{~b}$ ). For smaller values of $\tau$, the theory given above is not applicable. It follows from Fig. $4 \mathrm{a}$ 


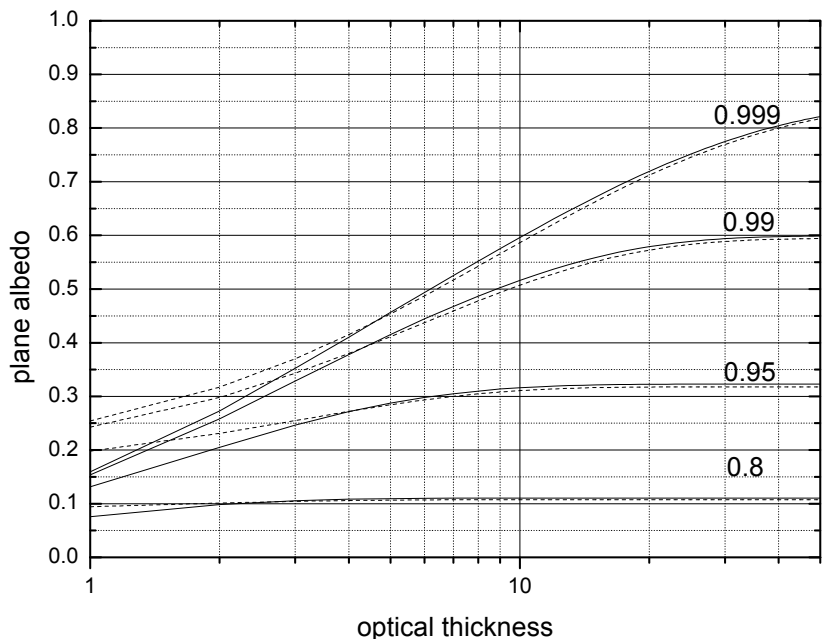

Fig. 4a. The same as in Fig. 1a except as a function of the optical thickness for a solar zenith angle of $60^{\circ}$. Approximate results are given by broken lines. Results obtained using SCIATRAN are represented by solid lines.

that the limit of the semi-infinite medium is reached at $\tau=5$ for a single scattering albedo equal to 0.8 (and, therefore, also at $\left.\omega_{0}<0.8\right)$.

The diffuse transmittance and also the diffuse absorptance calculated with the exact and approximate equations are shown in Fig. 5a as a function of the optical thickness. As one might expect the transmittance decreases and the absorptance increases with $\tau$. These physical dependencies are well represented by equations shown above at least till $\omega_{0}=0.8$ and the errors are generally smaller than $10 \%$ (see Figs. 5b, c). Comparatively large errors for $t_{d}$ at $\omega_{0}=0.8$ are due to the fact that $t_{d} \ll 1$ in this case.

Equations for $r_{p}$ and $t_{d}$ are simplified at $\omega_{0}=1$. Then it follows (Kokhanovsky, 2006):

$r_{p}=1-t_{d}, t_{d}=\frac{K_{0}\left(\mu_{0}\right)}{1.072+0.75 \tau(1-g)}$.

where $K_{0}\left(\mu_{0}\right) \equiv K\left(\mu_{0}, \omega_{0}=1\right)$. The accuracy of Eqs. (17) is studied in Figs. 6, 7 for the same phase function as has been used above and assuming that (Kokhanovsky, 2006)

$K_{0}\left(\mu_{0}\right)=\frac{3}{7}\left(1+2 \mu_{0}\right)$.

This equation gives the parameterization of the correspond LUT at $\omega_{0}=1$ and $\mu_{0} \geq 0.2$.

The analysis of Figs. 6-7 shows that the simple Eq. (17) can be used for the computation of $r_{p}$ and $t_{d}$ with an error smaller than 5\% at $\tau \geq 7$ and $\tau \geq 5$ respectively. For both parameters, the error is smaller than $1 \%$ at $\tau \geq 10, \omega_{0}=1$.

It is evident that errors for the pair $\left(r_{s}, t\right)$ are even smaller than those for $r_{p}$ and $t_{d}$. This is mostly due to the fact that one more integration is involved in the definition of $\left(r_{s}, t\right)$ as

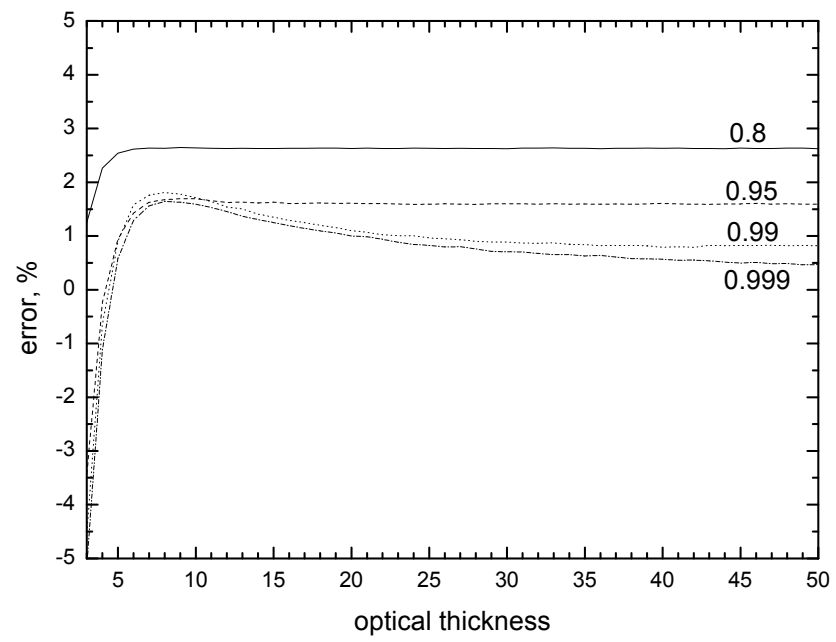

Fig. 4b. Errors calculated using data shown in Fig. 4a.

compared to $\left(r_{p}, t_{d}\right)$. In particular, we have found that the error of the asymptotic theory is smaller than $2 \%$ at $\tau \geq 3$ for $r_{s}$ and it is smaller than 5\% at $\tau \geq 5$ for $t$ at $g=0.85$. The error decreases further with cloud optical thickness.

\subsection{Reflection function}

The accuracy of Eqs. (1), (2) is studied in Fig. 8 for $A=0$ and several values of $\omega_{0}$ for the same phase function as above $(g=0.85)$. Equations (1), (2) are transformed to the following forms at $\omega_{0}=1$ :

$$
\begin{aligned}
& R_{A}\left(\mu_{0}, \mu, \phi\right)=R\left(\mu_{0}, \mu, \phi\right)+\frac{A t_{d}\left(\mu_{0}\right) t_{d}(\mu)}{1-A r_{s}}, \\
& R\left(\mu_{0}, \mu, \phi\right)=R_{\infty 0}\left(\mu_{0}, \mu, \phi\right)-T\left(\mu_{0}, \mu\right), \\
& T_{A}\left(\mu_{0}, \mu\right)=T\left(\mu_{0}, \mu\right)+\frac{A t_{d}\left(\mu_{0}\right) r_{p}(\mu)}{1-A r_{s}}, \\
& T\left(\mu_{0}, \mu\right)=t K_{0}\left(\mu_{0}\right) K_{0}(\mu),
\end{aligned}
$$

where $R_{\infty 0}\left(\mu_{0}, \mu, \phi\right) \equiv R_{\infty}\left(\mu_{0}, \mu, \phi, \omega_{0}=1\right)$ and

$$
t=\frac{1}{1.072+0.75 \tau(1-g)}
$$

is the global transmittance at $\omega_{0}=1$. Due to the conservation of energy principle it follows for the spherical albedo of nonabsorbing media: $r_{s}=1-t$.

The comparison of exact and approximate results for a nadir observation and solar zenith angles ranging from 0 to 85 degrees is shown in Fig. 8 for the special case of a black underlying surface. It follows that the approximations and exact results are very close to each other at $\omega_{0}>0.8$ (the relative error is smaller than $2 \%$ ). Smaller values of $\omega_{0}$ are not typical for cloudy media in the visible and near-infrared. The high accuracy of the expression for $R$ confirms that the value 


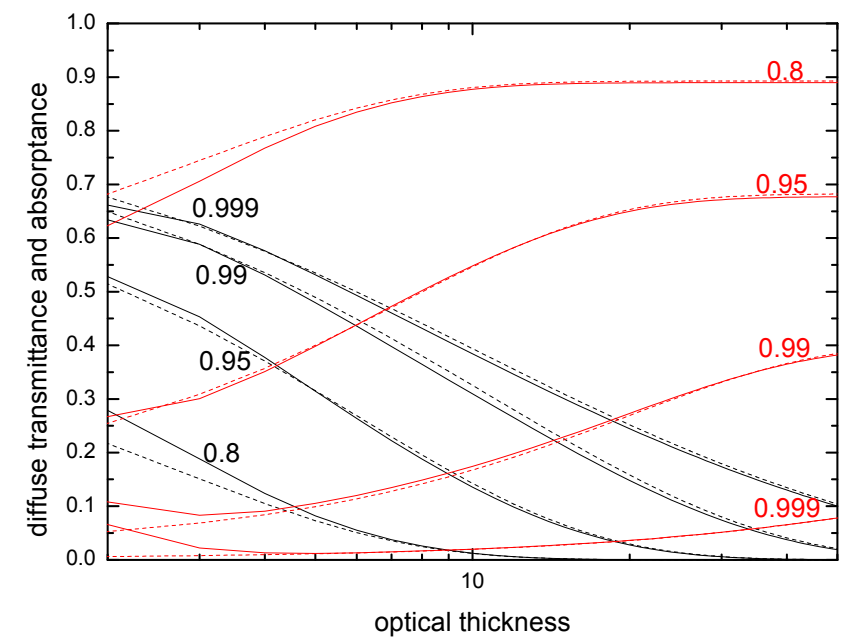

Fig. 5a. Dependence of the diffuse transmittance (black lines) and absorptance (red lines) on the optical thickness for several values of the single scattering albedo and an incidence zenith angle of $60^{\circ}$. Approximate results are given by broken lines.

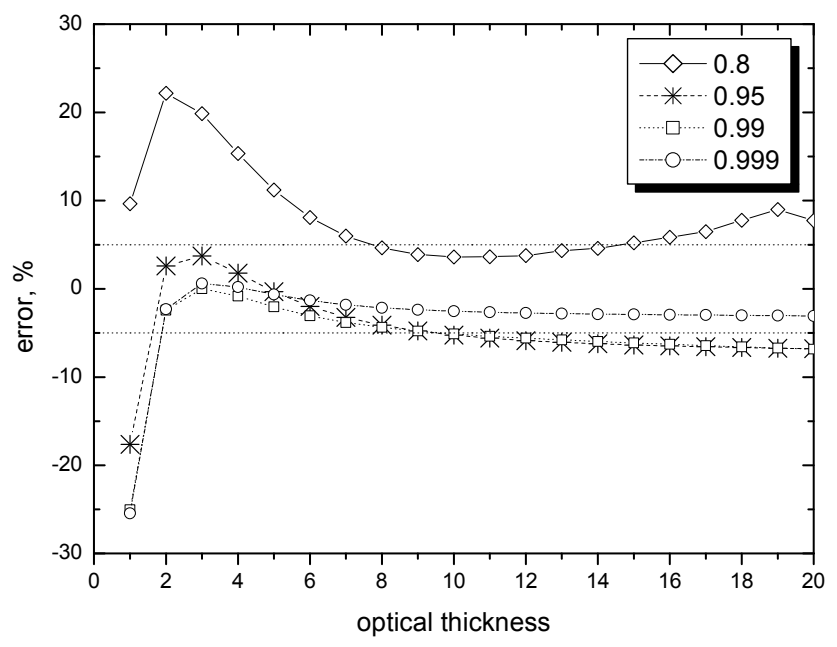

Fig. 5b. Errors in $t_{d}$ calculated using data shown in Fig. 5a.

of $T=\left[R_{\infty}\left(\mu_{0}, \mu, \phi\right)-R\left(\mu_{0}, \mu, \phi\right)\right] l^{-1} e^{k \tau}$ is also calculated accurately in the framework of the approximation considered here.

Another interesting aspect is the behavior of the approximations for non-nadir observations. The results of calculations for an observation angle of 60 degrees and several azimuth angles are shown in Fig. 9. One can see that the approximation is capable to describe the case of non-nadir observations. For instance, the glory seen on the exact curve at an azimuth of 180 degrees is well reproduced by the approximation. Errors are below $0.5 \%$ for the case shown in Fig. 9.

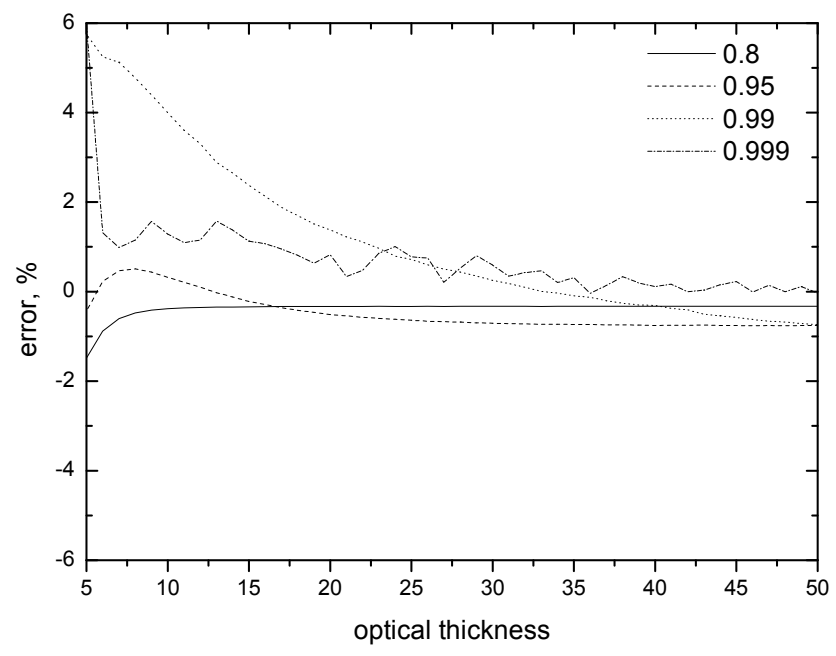

Fig. 5c. Errors in $a_{d}$ calculated using data shown in Fig. 5a.

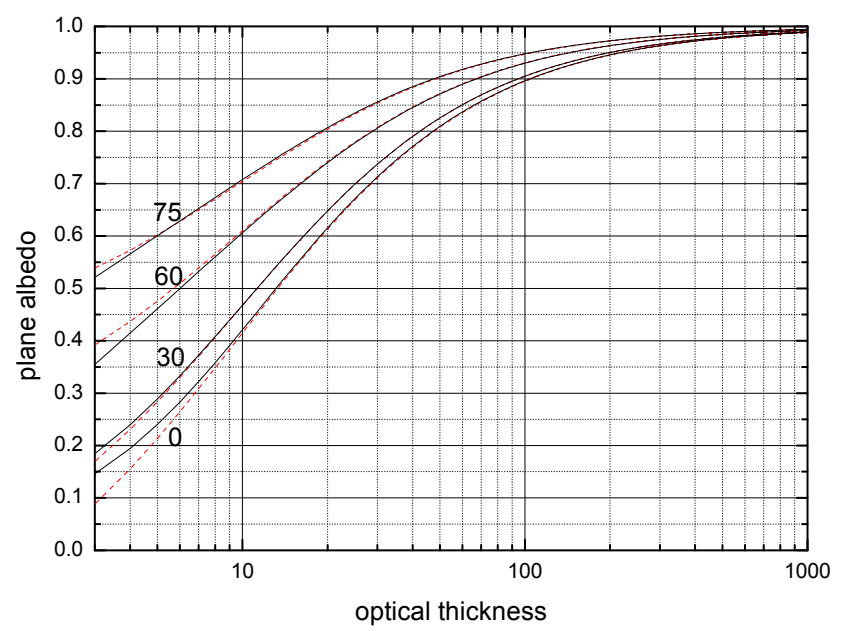

Fig. 6a. Dependence of the plane albedo on the optical thickness for several solar angles and $\omega_{0}=1$ according to exact results(black lines) and approximations (red lines).

Clearly, errors increase for smaller $\tau$. This is shown in Fig. 10. However, they remain smaller than $5 \%$ at $\tau \geq 5$ (see Fig. 10b) and smaller than $1 \%$ at $\tau \geq 10$ and $\omega_{0} \geq 0.8$. Smaller values of $\omega_{0}$ are of no interest because such low values are not characteristic for water clouds in the visible and nearinfrared. Interestingly, the approximation errors decrease with $\beta=1-\omega_{0}$ for a given $\tau$. In particular, the code can be used with an error smaller than $5 \%$ at $\tau \geq 2$ and $\omega_{0}=0.8$ and errors are smaller than $5 \%$ at $\tau \geq 3$ and $\omega_{0} \leq 0.95$. For nonabsorbing clouds, the errors are smaller than $5 \%$ at $\tau \geq 5$ (see Fig. 10b).

All results shown above have been obtained for the case of clouds with droplet size distributions having an effective radius $6 \mu \mathrm{m}$. It is of importance to see how the equations 


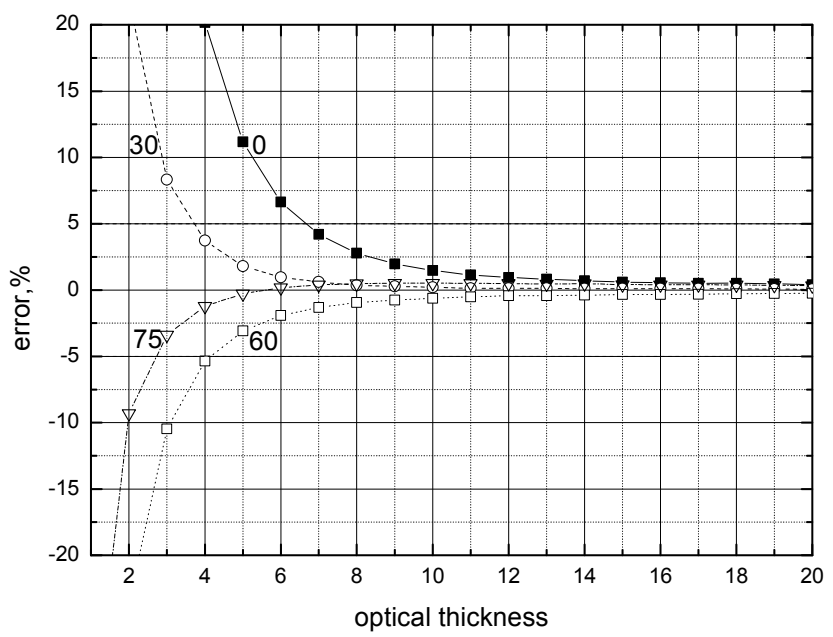

Fig. 6b. Errors calculated using data shown in Fig. 6a.

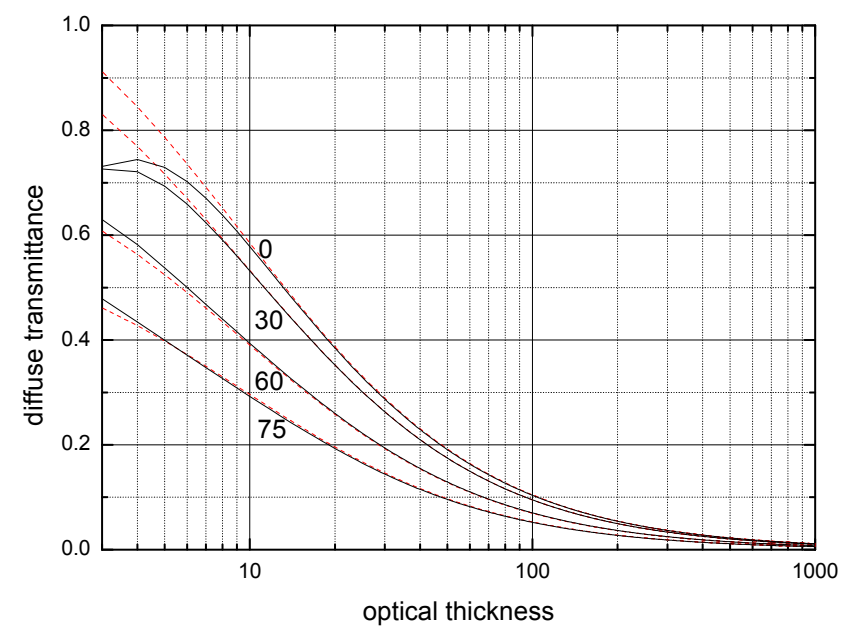

Fig. 7a. The same as in Fig. 6a except for the diffuse transmittance.

behave if the exact calculations are obtained for a different value of the effective radius as compared to that used in the preparation of LUTs. Therefore, Fig. 11 shows a comparison for the case of $\omega_{0}$ equal to $0.95,0.99$ and 1.0 between exact results computed for an effective radius of $16 \mu \mathrm{m}$ and the approximations that are based on LUT values valid for an effective radius of $6 \mu \mathrm{m}$. It follows that the errors for nonabsorbing clouds generally stay within $5 \%$. They are somewhat larger in the vicinity of the rainbow and glory region. This is related to the fact that the position of the rainbow and also the angular positions of the glory rings are different for 6 and $16 \mu \mathrm{m}$ droplets. This problem will not arise for ice clouds because in this case, both rainbows and glories are absent. This can be seen in Fig. 12, which shows the comparison of the asymptotic results with exact calculations for ice clouds with fractal particles. The reflection functions of the

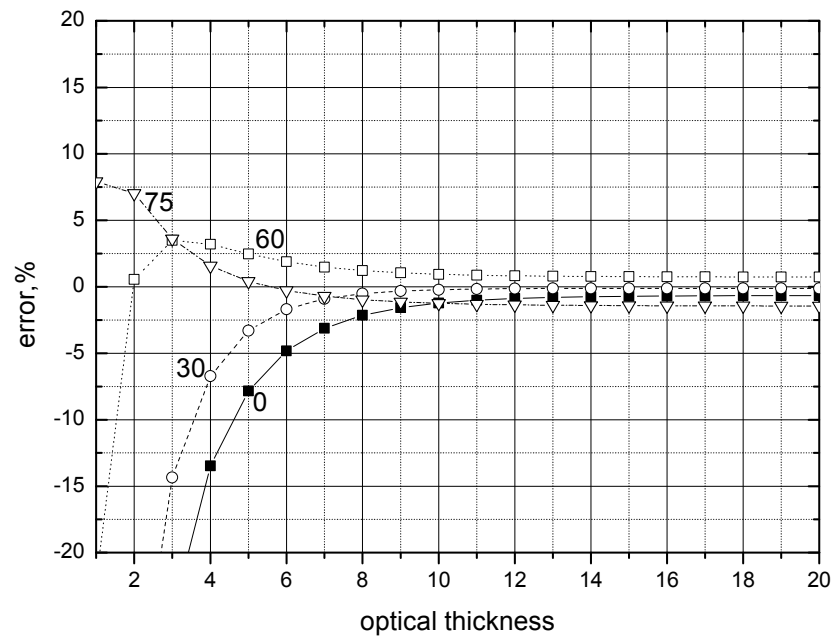

Fig. 7b. Errors calculated using data shown in Fig. 7a.

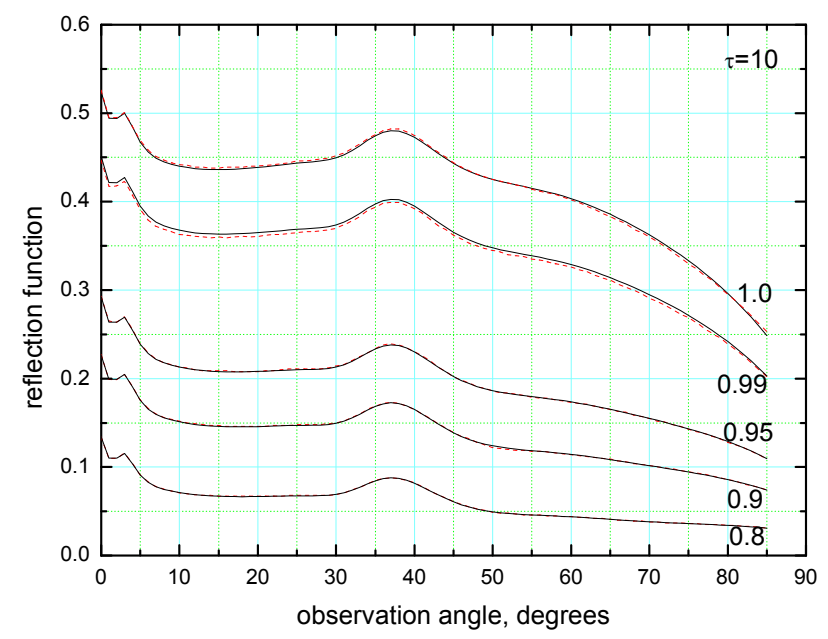

Fig. 8. Dependence of the reflection function on the incidence zenith angle for several values of the single scattering albedo and an optical thickness equal to 10 . Exact results are given by black lines. Approximate results are given by red broken lines.

ice clouds are very smooth and do not have a glory or rainbow angular features. The different behavior between water and ice clouds in this angular region could be used for the cloud phase discrimination using, e.g., azimuthally scanning optical instruments. Generally, errors for ice clouds $(\mathrm{g}=0.75)$ are also small ( $\leq 2 \%$ for most of angles).

\section{Conclusions}

The accuracy of a number of analytical equations for cloud optical characteristics including reflection and transmission function over a reflective Lambertian ground has been studied. The asymptotic theory can be used for rapid estimations 


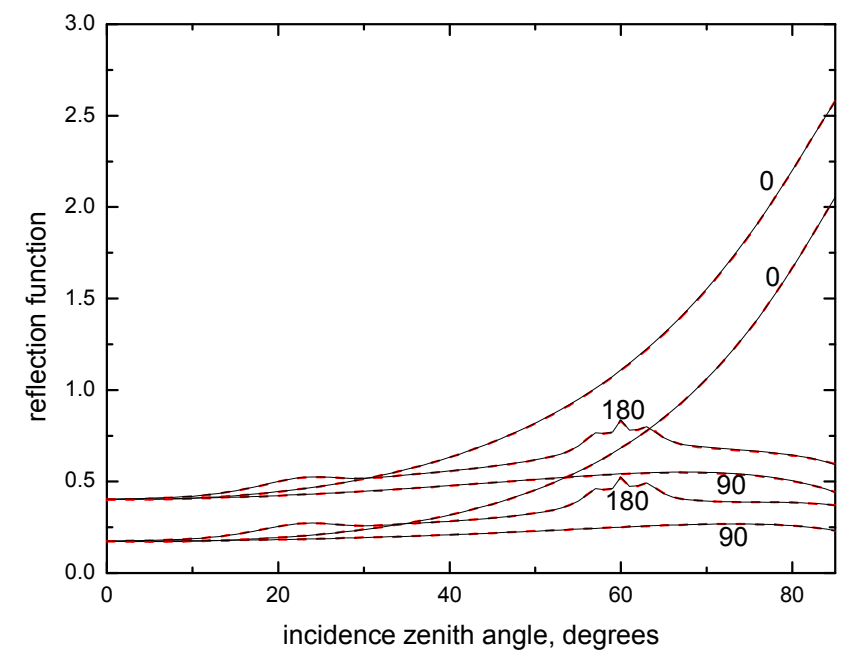

Fig. 9. Dependence of the reflection function on the incidence zenith angle for values of the single scattering albedo equal to 0.95 and 1.0, an optical thickness equal to 10 , an observation angle of $60^{\circ}$ and azimuth angles of $0^{\circ}, 90^{\circ}$, and $180^{\circ}$. Lower lines correspond to the smaller values of $\omega_{0}$. Red broken lines show approximate results. Solid lines present exact radiative transfer calculations. The correspondent absolute value of the relative error $|\delta|$ is smaller than $0.5 \%$.

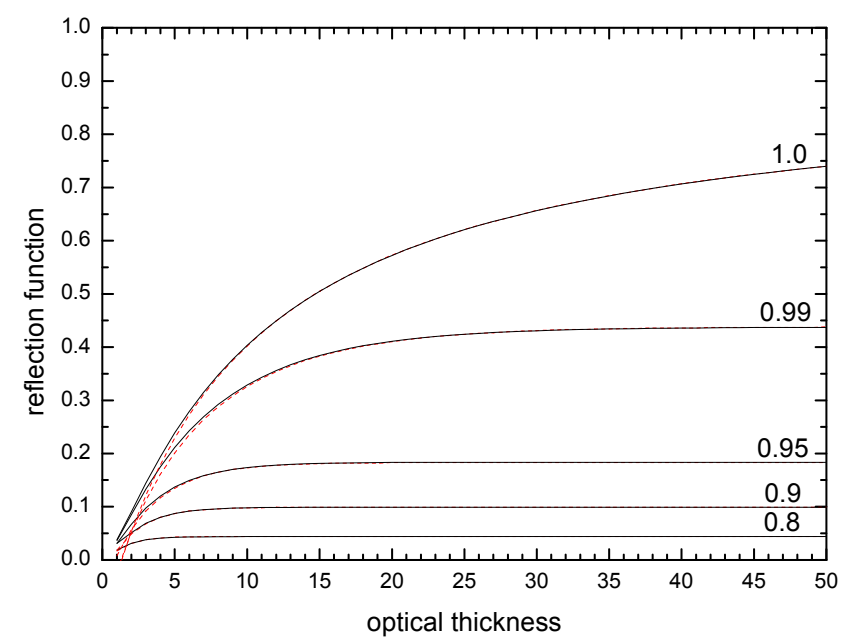

Fig. 10a. Dependence of the reflection function on the optical thickness for values of the single scattering albedo equal to $0.8,0.9,0.95$, $0.99,1.0$, an observation angle of $0^{\circ}$ and an incidence angle of $60^{\circ}$. Red broken lines show approximate results. Solid lines present exact radiative transfer calculations.

of the radiative characteristics in cloudy atmospheres. It is accurate for a cloud optical thickness $\tau \geq 5$ (and for some radiative transfer characteristics (see Fig. 4a) at $\tau \geq 3$ ). Only properties of optically thick water and ice clouds can be studied in the framework of the approximate theory discussed in this study.

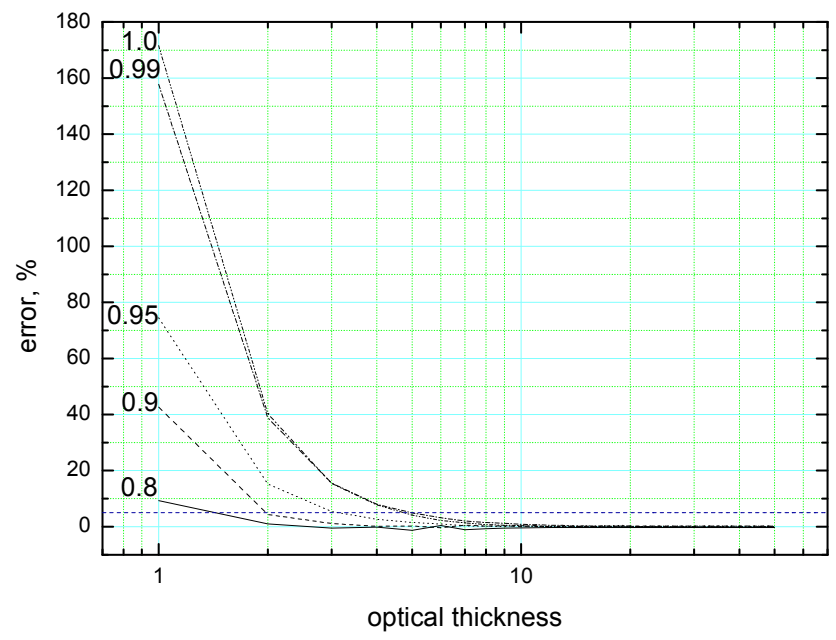

Fig. 10b. Dependence of the relative error on the optical thickness calculated using data shown in Fig. 10a for values of the single scattering albedo equal to $0.8,0.9,0.95,0.99,1.0$.

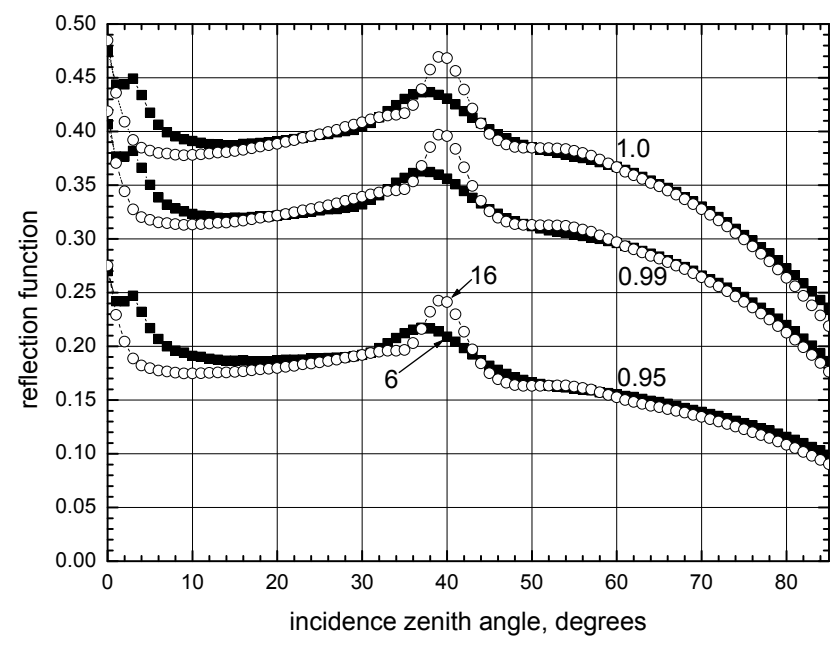

Fig. 11a. Dependence of the reflection function on the incidence zenith angle for values of the single scattering albedo equal to 0.95 , 0.99 and 1.0, an observation angle of $0^{\circ}$ and an incidence angle of $60^{\circ}$. Filled squares show approximate results. Circles give the results of the exact radiative transfer calculations. The increase of the error for the approximation in the rainbow and glory regions is due to the fact that LUTs for particles with an effective radius of $6 \mu \mathrm{m}$ have been used to derive the approximate results. Exact calculations have been performed for an effective particle radius of $16 \mu \mathrm{m}$. The asymmetry parameter $(\mathrm{g}=0.8692)$ has been the same for both calculations.

Asymptotic equations are very simple and can be used as a basis for the construction of high speed satellite cloud retrieval algorithms, which account for both multiple light scattering in a cloud and reflective properties of an underlying surface. They can be also used as a basis for the 


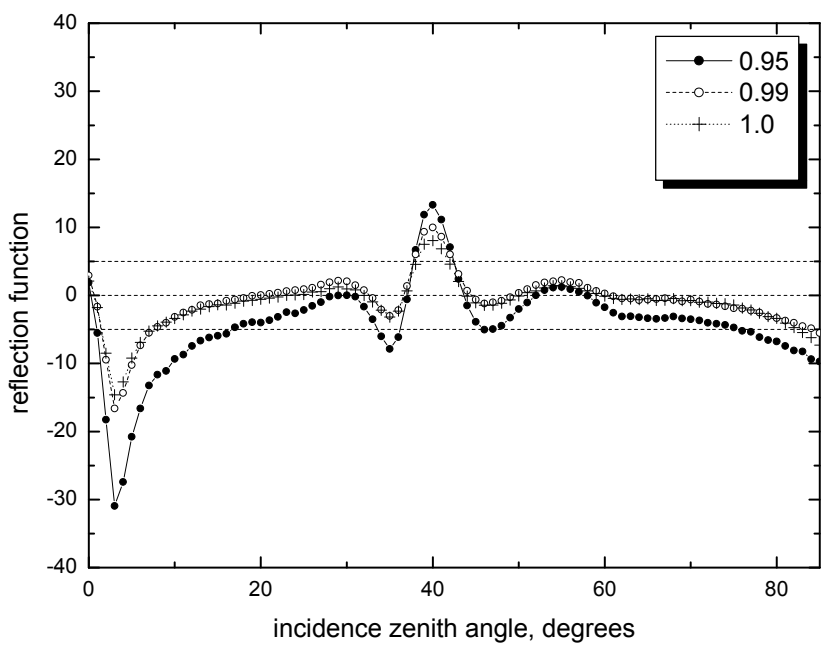

Fig. 11b. Dependence of the relative error on the incidence zenith angle calculated using data shown in Fig. 11a for values of the single scattering albedo equal to $0.95,0.99$ and 1.0 .

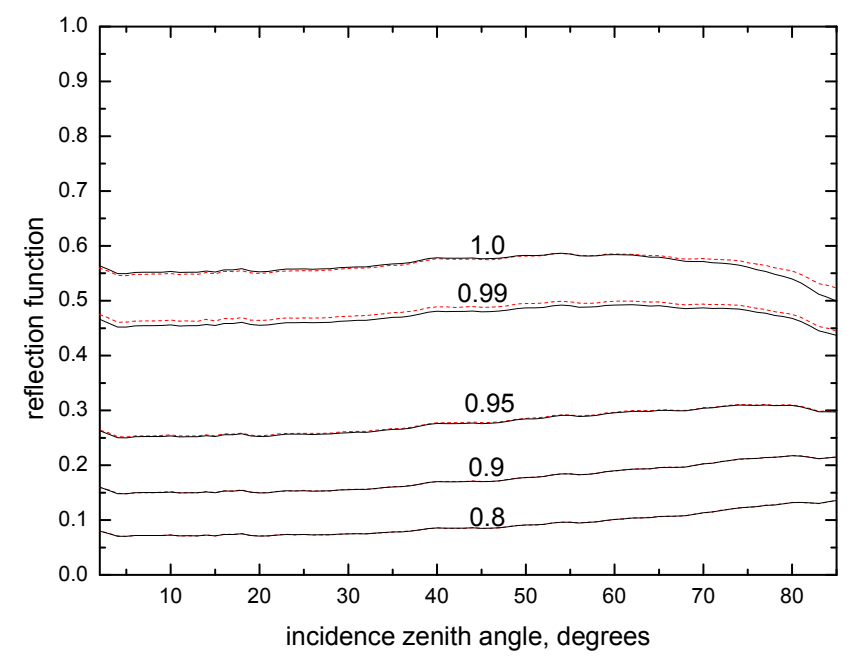

Fig. 12. Dependence of the reflection function on the incidence zenith angle for values of the single scattering albedo equal to 0.8 , $0.9,0.95,0.99$, and 1.0 , an optical thickness equal to 10 and an observation angle of $0^{\circ}$. Red broken lines show approximate results. Solid lines represent exact radiative transfer calculations. The correspondent absolute value of the relative error $|\delta|$ is smaller than $2 \%$ at $\vartheta_{0} \leq 75^{\circ}$. The fractal ice crystal phase function $(g=0.7524)$ as described by Mishchenko et al. (1999) has been used in the calculations.

parameterization of cloud retrieval LUTs for the case $\tau \rightarrow \infty$. The correspondent errors (for the case of thick clouds) are smaller than the uncertainties in the forward models (e.g., the assumption of plane-parallel homogeneous clouds) and they are below calibration errors of optical instruments currently in orbit.
The accuracy of the equations presented is dependent on the characteristic in question. However, it is smaller than 5\% for most of the studied cases relevant for the propagation of visible and near-infrared radiation in a cloudy atmosphere. Therefore, the equations could be used for the parameterization of radiative transfer blocks in global circulation and climate models, where even larger errors of approximations are commonly accepted due to computation time constrains and various other uncertainties inherent to atmospheric models.

The correspondent radiative transfer code CLOUD together with a user guide is available on line at http://www. iup.physik.uni-bremen.de/ alexk.

Acknowledgements. The authors would like to thank J. P. Burrows, V. V. Rozanov for their help and advice and W. M. F. Wauben as well as M. Mishchenko for providing the radiative transfer codes used for the computation of the LUTs. This work has been supported by the DFG Project DFG BU/688-2, the GLOWA-Danube project 97 GWK 04 "Rainfall Retrieval" and the Erich-Becker foundation (formerly Frankfurt Airport foundation), Germany.

Edited by: Q. Fu

\section{References}

King, M. and Harshvardhan: Comparative accuracy of selected multiple scattering approximations, J. Atmos. Sci., 44, 1734 1751, 1986.

Kokhanovsky, A. A., Rozanov, V. V., Zege, E. P., Bovensmann, H., and Burrows, J. P.: A semi-analytical cloud retrieval algorithm using backscattered radiation in $0.4-2.4 \mu \mathrm{m}$ spectral region, J. Geophys. Res., 108, 4008, doi:10.1029/2001JD001543, 2003.

Kokhanovsky, A. A.: Cloud Optics, Berlin: Springer-Verlag, 2006.

Mishchenko, M. I., Dlugach, J. M., Yanovitskij, E. G., and Zakharova, N. T.: Bidirectional reflectance of flat, optically thick particulate layers: an efficient radiative transfer solution and applications to snow and soil surfaces, J. Quant. Spectrosc. Radiat. Trans., 63, 409-432, 1999.

Nakajima, T. and King, M. D.: Determination of the optical thickness and effective particle radius of clouds from reflected solar radiation measurements, Part 1. Theory, J. Atmos. Sci., 47, 1878-1893, 1990.

Rozanov, A. V., Rozanov, V., Buchwitz, M., Kokhanovsky, A., and Burrows, J. P.: SCIATRAN 2.0: a new radiative transfer model for geophysical applications in the $175-2400 \mathrm{~nm}$ spectral region, Adv. in Space Res., 36, 1015-1019, 2005.

van de Hulst, H. C.: The spherical albedo of a planet covered with a homogeneous cloud layer, Astron. Astrophys., 35, 209-214, 1974.

van de Hulst, H. C.: Multiple Light Scattering: Tables, Formulas and Applications, N.Y., Academic Press, 1980.

Wauben, W. M. F.: Multiple Scattering of Polarized Radiation in Planetary Atmospheres, Ph.D. Thesis, Free University of Amsterdam, 1992. 